\title{
Investigation on crater morphology by high repetitive rate YAG laser-induced discharge texturing
}

\author{
Zhengyang $\mathrm{Li}^{\mathrm{a}}{ }^{\mathrm{b}, *}$, Yang Mingjiang ${ }^{\mathrm{b}}$, Wenjin Liu ${ }^{\mathrm{a}}$, Minlin Zhong ${ }^{\mathrm{a}}$ \\ ${ }^{a}$ Key Laboratory for Advanced Manufacturing by Materials Processing Technology, Department of Mechanical Engineering, \\ Tsinghua University, Beijing, 100084, PR China \\ ${ }^{\mathrm{b}}$ Institute of Mechanics, Chinese Academy of Sciences, Beijing, 100080, PR China
}

Received 28 October 2004; accepted in revised form 4 March 2005

Available online 31 May 2005

\begin{abstract}
An innovative texturing technique, high repetitive rate YAG laser-induced discharge texturing (LIDT), has been developed. The principle is a laser pulse synchronized with electrical pulse radiates on one of two electrodes, a plasma plume appears, that serves as discharge channel and the breakdown may occur between the electrodes far below the breakdown threshold. If the roll and a conical metal rod are the two electrodes, with the rotating of roll and the moving of laser set and conical metal rod along the axial of the roll, the deterministic or the random crater patterns are obtained on the roll surface by a serial laser-induced discharge. The experiments were carried out in different current discharge waveforms, media and electrical polarities on several materials. The results show that crater morphology is mainly determined by electrical polarity and medium. Simple explanations of the result are suggested.
\end{abstract}

(C) 2005 Elsevier B.V. All rights reserved.

Keywords: Laser; Surface morphology; Discharge; Texturing

\section{Introduction}

The surface texturing of steel sheets has become an important issue in steel production and its applications [1,2]. Textured roll mills the sheet and the morphology of roll is copied to the sheet. There are many techniques used to texture roll surface, such as shot blasting (SB), $\mathrm{CO}_{2}$ laser texturing, electrical discharge texturing (EDT) and electronic beam texturing (EBT) [3-6]. High repetitive rate YAG laser pulse texturing, first developed by Institute of Mechanics, Chinese Academy of Sciences in 1992 [7-10], is also one of the surface texturing techniques. According to crater patterns obtained, the above texturing techniques are usually classified as the deterministic and the random. Laser texturing and EBT are the deterministic, and SB and EDT are the random. The two patterns have their advantages and disadvantages according to different applications [11-13]. It

\footnotetext{
* Corresponding author.

E-mail address: alxzyli@cei.gov.cn (Z. Li).
}

is also found that the techniques mentioned above not only change surface morphology, but also harden the surface to increase roll's service life. But the depth of hardening zone by laser texturing is relatively low, for the YAG laser texturing it is only several dozen micrometers. To obtain deep hardening zone and a deterministic pattern, it is necessary to increase the pulse energy and the density of pulse power. Increasing laser power is one of the solutions, but the repetitive rate of laser pulse has to be decreased. Another disadvantage of laser is its absorptivity by metal is low. Though EDT may deepen hardening zone, its crater pattern is random. The crater pattern of EBT is deterministic and the absorptivity of electronic beam by metal is high, but the processing must be carried out in the vacuum.

To take advantages of the controllability of laser and the high electrical absorptivity by metal, an innovative texturing technique, high repetitive rate YAG laser-induced discharge texturing (LIDT), is developed. The application of laser induced air breakdown has been extensively investigated [14-16]. Though laser-induced discharge has been used in boring hole and image marking $[17,18]$, its use to texture 
metal surface has not been reported. Since the surface morphology, i.e. the patterns and the shape of crater, are major concerns of surface texturing, the present work focuses on crater morphology of LIDT on several materials with different media, discharge waveforms and electrical polarities. Simple explanations of the result are suggested. The hardness, the depth of hardening zone and the phase transformation of crater with various parameters in the processing will be reported in another paper.

\section{Experiment}

When an electrical pulse applies on the two electrodes in air, whose clearance is proximately $0.5 \mathrm{~mm}$, no breakdown will occur if the pulse peak is about several hundred volts. If a laser pulse synchronized with electrical pulse radiates on one of two electrodes, a plasma plume appears, that serves as discharge channel and the breakdown may occur between the electrodes far below the breakdown threshold. The duration of electrical pulse is far longer than that of laser pulse. The discharge is triggered by laser pulse and is shut off by the electrical pulse that is controlled by the IGBT (Insulated gate bipolar transistor) of the power supply. If the roll is as one of two electrodes and a conical metal rod as the other, discharge crater will be distributed on the roll by a serial laser-induced discharge with roll rotating and the moving of laser set and the conical metal rod along the axial of the roll (Fig. 1). With hardware and software controlling, deterministic or random crater patterns will be obtained easily (Fig. 2).

The experiment was carried out in air, but the surface of roll might be covered by a thin oil film sometimes, that meant the discharge media between the two electrodes were air or air and oil. The oil was engine oil and the thickness of oil film was about half clearance between two electrodes. The tip angle of conical electrode was $20^{\circ} \sim 45^{\circ}$. The rolls were made of steel (AISI 1045), cast iron and brass, respectively, and the conical electrode was made of $\mathrm{CuW}$. The clearance between the two electrodes was $200 \sim 300 \mu \mathrm{m}$. The laser pulse was modified by a Q-switch on a 150 watts

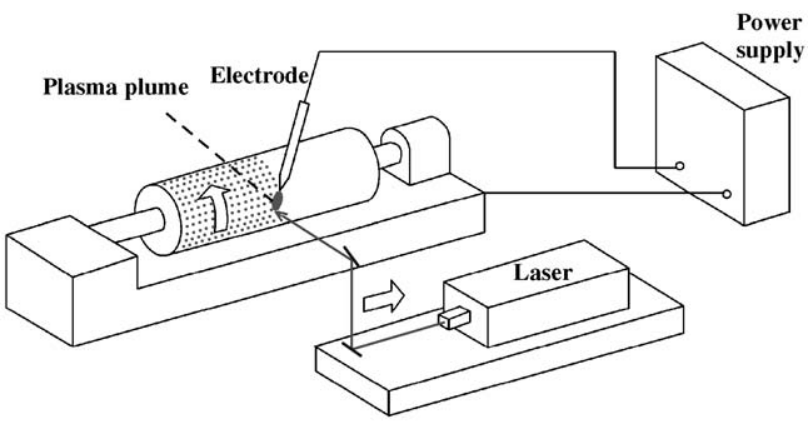

Fig. 1. The principle of high repetitive rate YAG laser-induced discharge texturing.

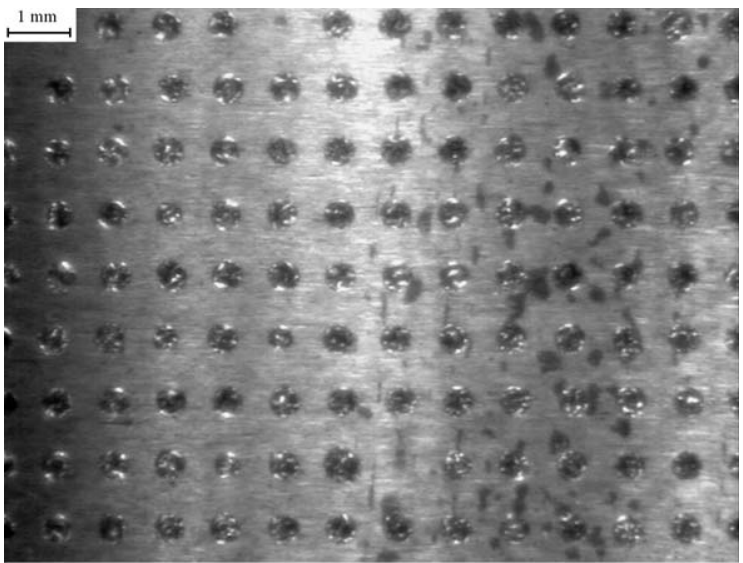

(a) Deterministic pattern of craters

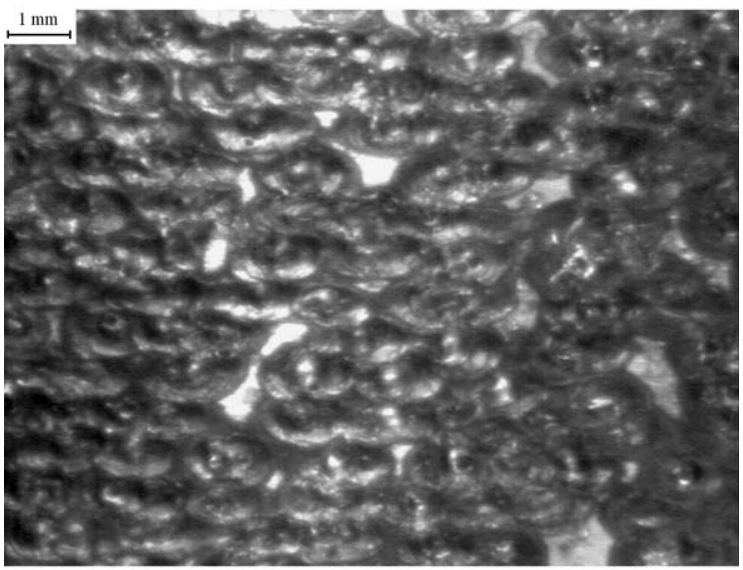

(b) Random pattern of craters

Fig. 2. Crater patterns of laser-induced discharge texturing.

Nd: YAG laser oscillator. To be safe, the roll was always connected to the ground, but the electrical polarities between the roll and the conical electrode were alternated that meant the roll could be either cathode or anode. There were two current discharge waveforms by adjusting parameters of power supply. One was triangle (Fig. 3(a) curve $C$ ), another was rectangle (Fig. 3(b) curve $C$ ). It was found that voltage waveforms remained constant after discharging (curve $B$ at Fig. 3(a) and Fig. 3(b)). To compare with different discharges, the energy of each discharge pulse was set to be the same as 1 J. $E=\int_{0}^{\tau} i u t$, where $i$ and $u$ were current and voltage, respectively, and were recorded by Tektronix TDS 210 oscillograph, $\tau$ was pulse duration. The discharge craters were cut from the roll and observed by PolyvarMET microscope. The circumference of crater was framed by a rectangle on the screen of microscope, the periphery of rectangle was measured and divided by 4 as an equivalent diameter of the crater.

\section{Results}

The crater morphology of different roll materials, electrical polarities and media is shown from Figs. 4-9. 
(a) Triangular waveform

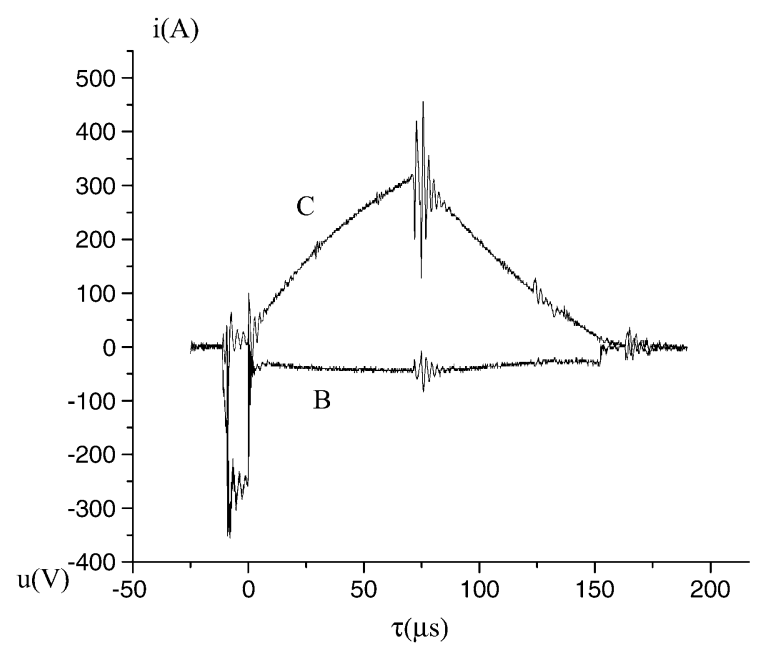

(b) Rectangular waveform

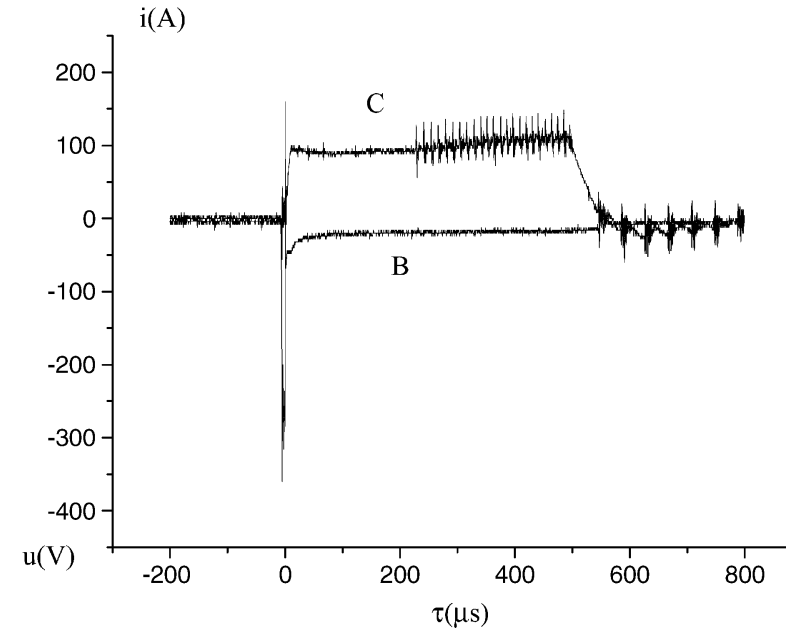

Fig. 3. Discharge waveforms. $B$ is voltage waveform, $C$ is current waveform. High negative volts $(300 \sim 350 \mathrm{~V})$ at $\tau=0$ in (a) and (b) are the voltage applied before breakdown.

The above figures show that a crater is constructed by many small craters and wave-like structures in most cases. We named the craters as multi-craters. The circumference of these craters is not very clear especially when they are used as cathode in air. It becomes very clear only in one case that the roll is used as anode and covered by a thin oil film no matter which materials - steel, iron or brass - is used. These craters are named as single-crater.

A phenomenon must be noticed that crater and laser spot may not be coincident in certain conditions when discharging in air (see arrows appoint in Fig. 4(c), Fig. 7(c), Fig. 9(a) and (c)). The phenomenon does not appear if the rolls are covered by a thin oil film. That means crater overlaps the laser spot and the laser induces a discharge successfully.

Current waveforms have little effect on crater morphology. They could not be discerned only by the crater morphology.
The diameters of crater are shown on Table 1. Comparing with the rows adjacent, for instance, row 1 and row 2 , row 5 and row 6 , etc., it can be concluded that

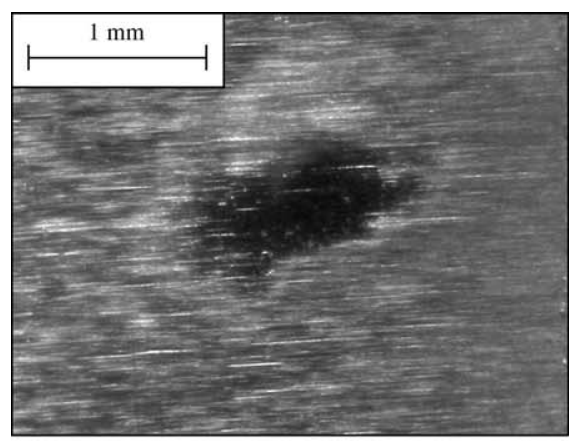

(a) Air and triangular waveform

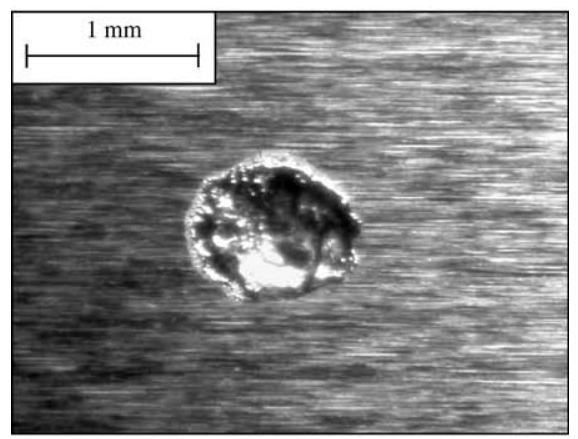

(b) Oil film and triangular waveform

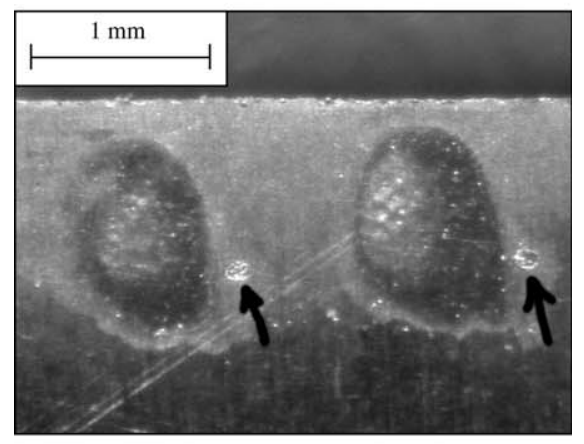

(c) Air and rectangular waveform

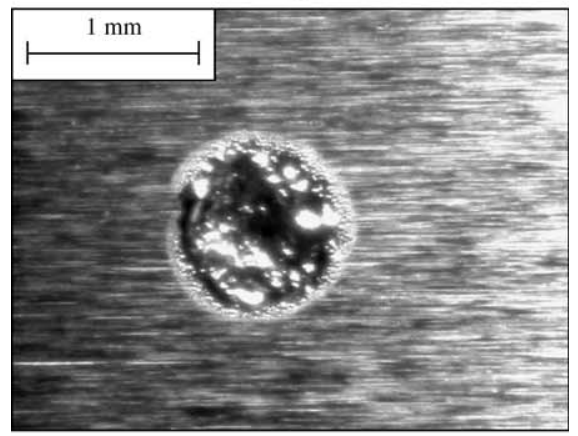

(d) Oil film and rectangular waveform

Fig. 4. Crater morphology of steel roll which was used as a cathode in different discharge media and current waveforms. (a), (b), (c), (d) are different combinations of media and waveforms. 


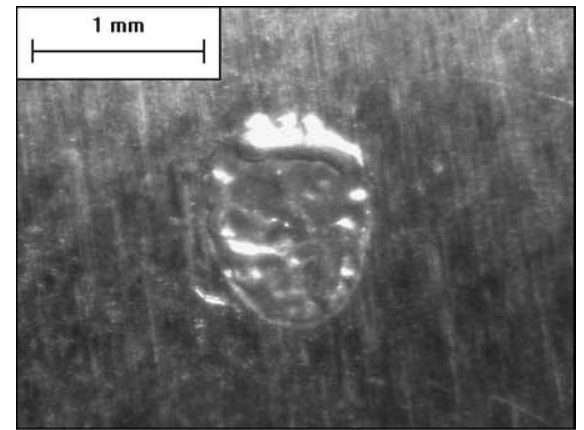

(a) Air and triangular waveform

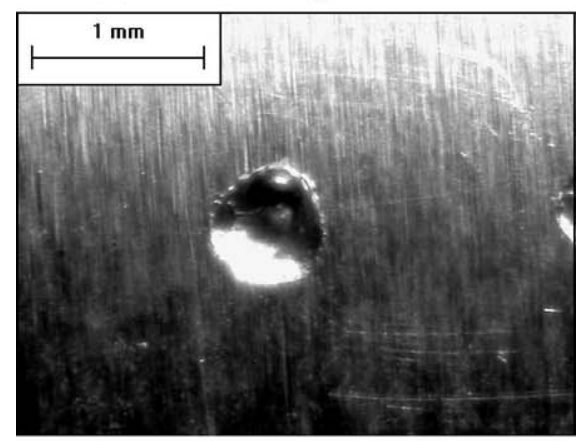

(b) Oil film and triangular waveform

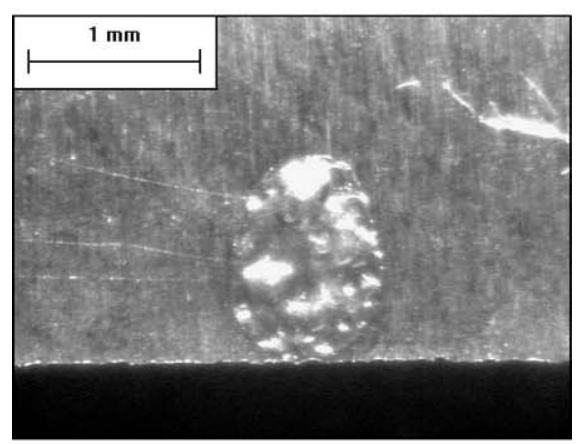

(c) Air and rectangular waveform

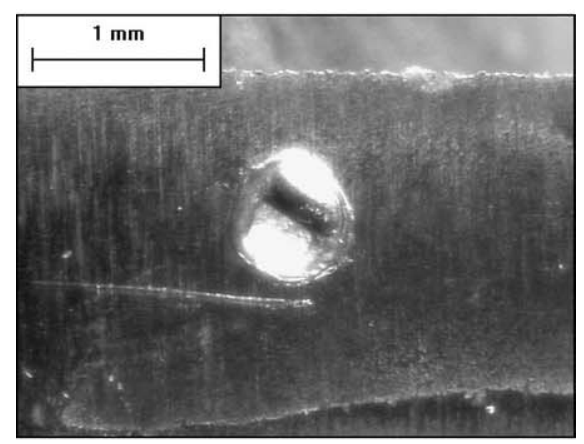

(d) Oil film and rectangular waveform

Fig. 5. Crater morphology of steel roll which was used as an anode in different discharge media and current waveforms. (a), (b), (c), (d) are different combinations of media and waveforms.

the diameter of crater discharging in air are always larger than that of in air and oil when other conditions are the same.

\section{Discussion}

\subsection{About multi-craters formation}

When discharging pulse duration is over $1 \mu \mathrm{s}$, the plasma in discharge channel reaches to a thermal equilibrium

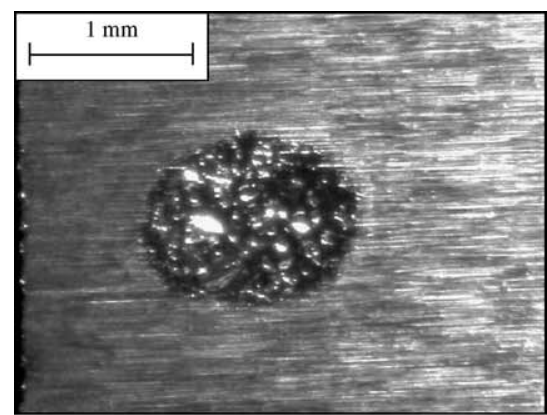

(a) Air and triangular waveform

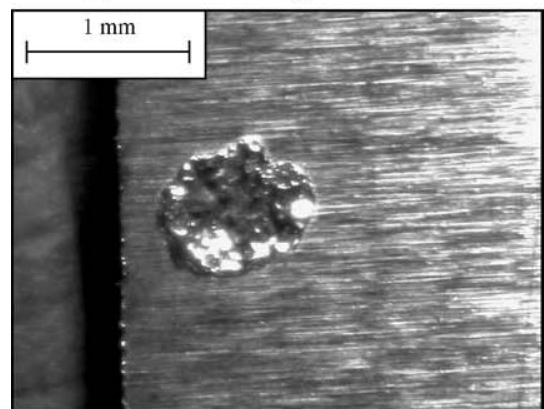

(b) Oil film and triangular waveform

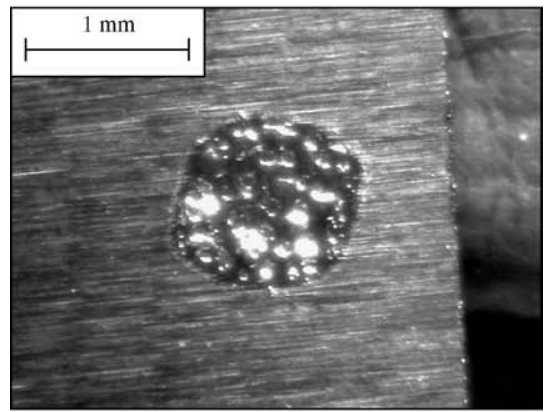

(c)Air and rectangular waveform

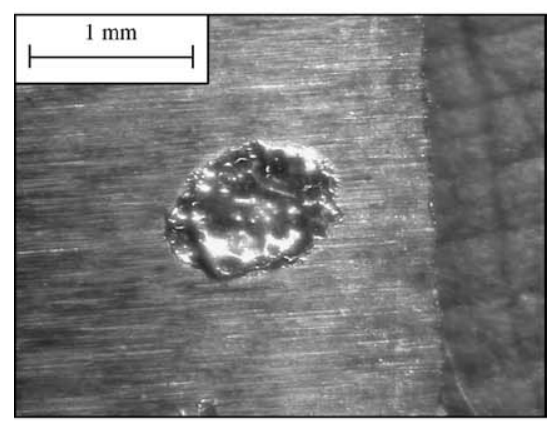

(d) Oil film and rectangular waveform

Fig. 6. Crater morphology of iron roll which was used as a cathode in different discharge media and current waveforms. (a), (b), (c), (d) are different combinations of media and waveforms. 


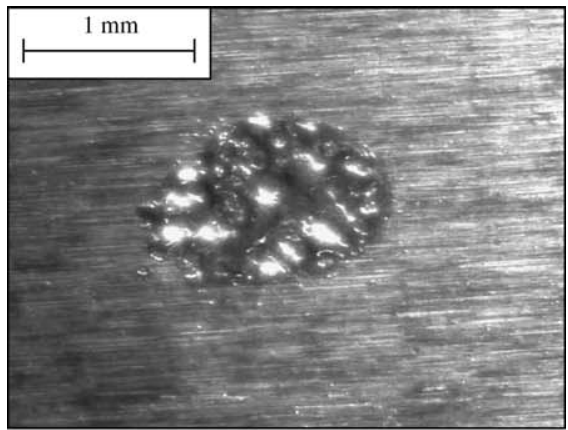

(a) Air and triangular waveform

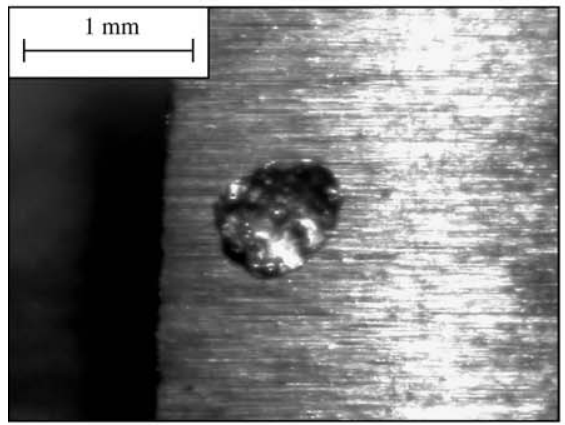

(b) Oil film and triangular waveform

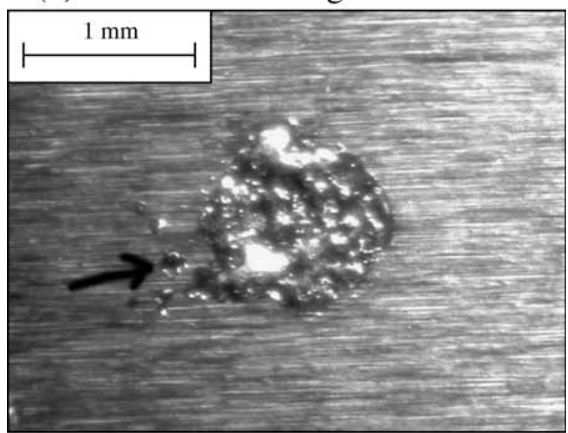

(c)Air and rectangular waveform

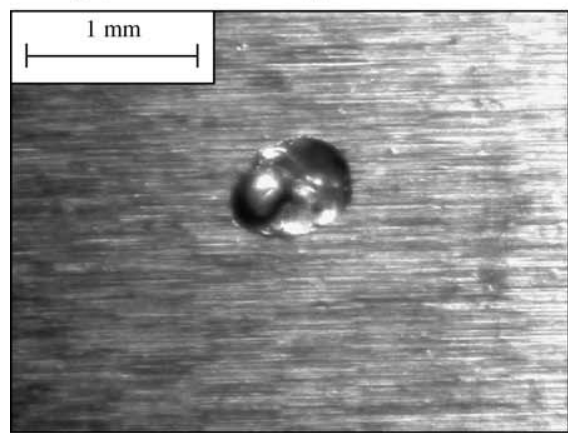

(d) Oil film and rectangular waveform

Fig. 7. Crater morphology of iron roll which was used as an anode in different discharge media and current waveforms. (a), (b), (c), (d) are different combinations of media and waveforms.

$[19,20]$. On the other hand, no matter which current waveform is used, the voltage maintains $30 \sim 40$ volts (see curve B in Fig. 3(a) and (b)) that is a distinguishing feature of arc discharge. Thus laser-induced discharge in our experiment can be treated as arc discharge. It is well known that arc column is pinched on cathode and is scattered on anode. If the cathode is cool, fast moving spot will appear. In the experiment, all conditions are fitted with the cool cathode, thus we can conclude the multi-craters on cathode are due to fast moving spots.

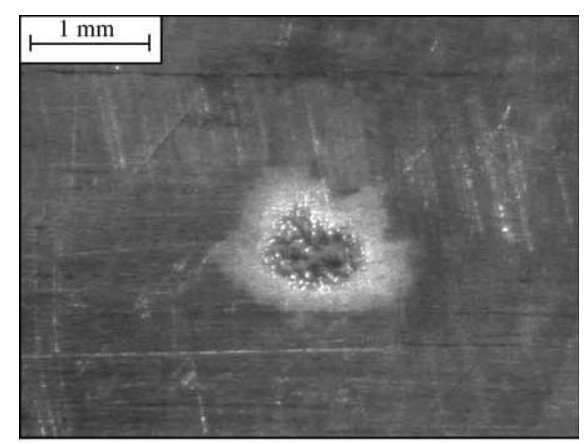

(a) Air and triangular waveform

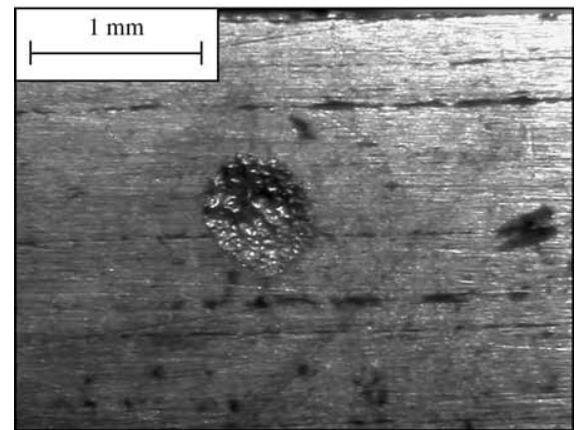

(b) Oil film and triangular waveform

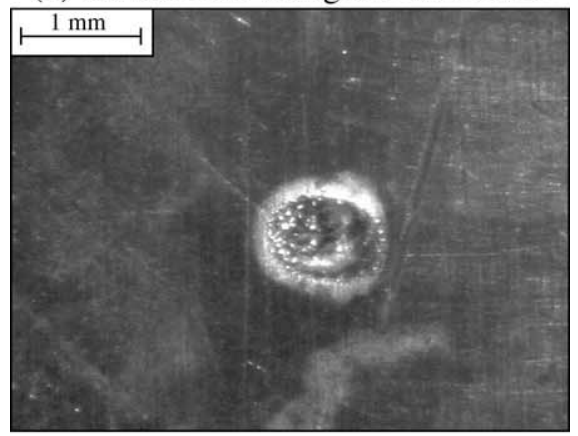

(c)Air and rectangular waveform

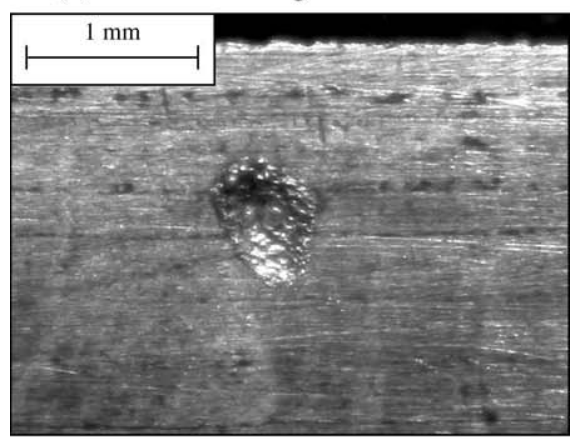

(d) Oil film and rectangular waveform

Fig. 8. Crater morphology of brass roll which was used as a cathode in different discharge media and current waveforms. (a), (b), (c), (d) are different combinations of media and waveforms. 


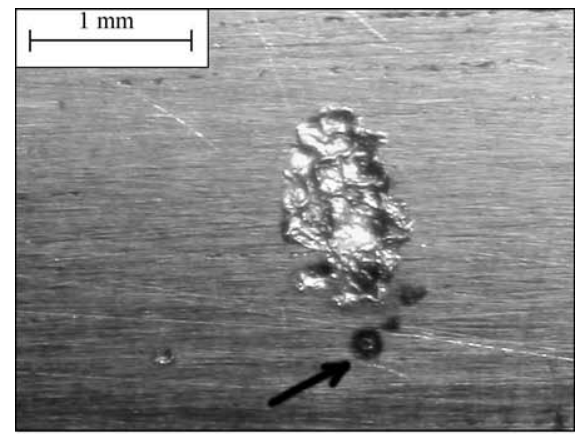

(a) Air and triangular waveform

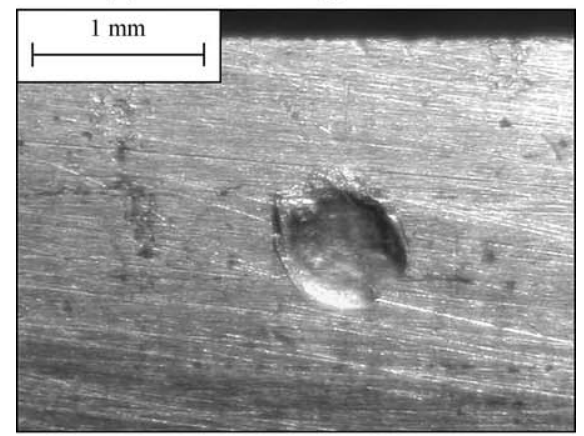

(b) Oil film and triangular waveform

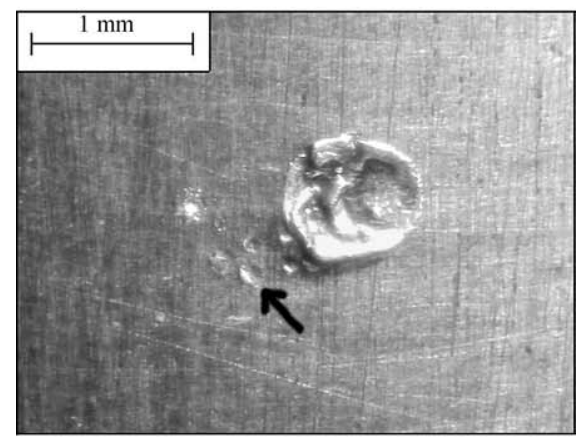

(c) Air and rectangular waveform

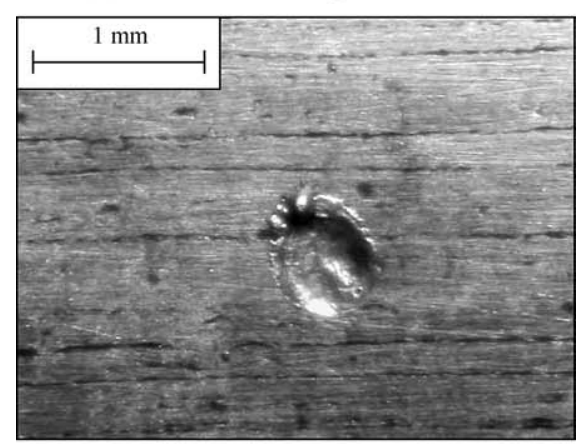

(d) Oil film and rectangular waveform

Fig. 9. Crater morphology of brass roll which was used as an anode in different discharge media and current waveforms. (a), (b), (c), (d) are different combinations of media and waveforms.

There may be two mechanisms about multi-craters formation on anode. Firstly anode spot is more stable than cathode spot, its velocity is also slow. The multi-craters may be formed by scattered pulse that is the result of plasma oscillation $[21,22]$. But we suggest here another mechanism
Table 1

Crater diameters of different materials with different electrical polarities, discharge media and current waveforms at the same electrical pulse energy $(\mu \mathrm{m})$

\begin{tabular}{llrrl}
\hline \multirow{2}{*}{ Discharge conditions } & \multicolumn{3}{l}{ Materials } & \\
\cline { 3 - 5 } & & Steel & Iron & Brass \\
\hline 1 & Air, anode and rectangular waveform & 1040 & 1088 & 824 \\
2 & Oil, anode and rectangular waveform & 801 & 718 & 697 \\
3 & Air, anode and triangular waveform & 1128 & 1232 & 983 \\
4 & Oil, anode and triangular waveform & 716 & 686 & 783 \\
5 & Air, cathode and rectangular waveform & $1000^{*}$ & 1075 & 765 \\
6 & Oil, cathode and rectangular waveform & 937 & 924 & 658 \\
7 & Air, cathode and triangular waveform & $1000^{*}$ & 1188 & 751 \\
8 & Oil, cathode and triangular waveform & 916 & 946 & 694 \\
\hline
\end{tabular}

Each data in the table is an average of 5 measured data.

* Since the circumference of crater is blurring in the cases, we use 1000 $\mu \mathrm{m}$ as an approximate value.

that may be more important for the formation of multicraters structures on anode if the feature of laser-induced discharge is considered. When a laser beam radiates on the surface of roll, metal plasma plume appears, in which many electrons is contained. The streamers are able to develop anywhere simultaneously or successively in the plasma between the roll and the conical electrode. The probability of discharge channel formation is great (Fig. 10), each discharge channel may form an anode spot, that results in multi-craters on anode. We call the mechanism as multichannels discharging.

\subsection{About single-crater formation}

If the roll is as anode and covered by a thin oil film, laser radiates on the surface of roll through the oil film, the plasma expanding is restricted by oil film in transverse (perpendicular to the laser beam), but almost free in longitudinal because the end of oil film opens to air and part of the plasma is still in air (Fig. 11), only the streamers developing along the laser beam result in discharge channels finally. The probability of channel formation decreases sharply. After discharge channel is formed, its expansion is still strongly restricted by the oil film near the surface of roll in transverse because the density and the thermal conduction of oil are greater than those of air.

The plasma channel with high pressure and high temperature is now focused on the laser-radiated spot. The

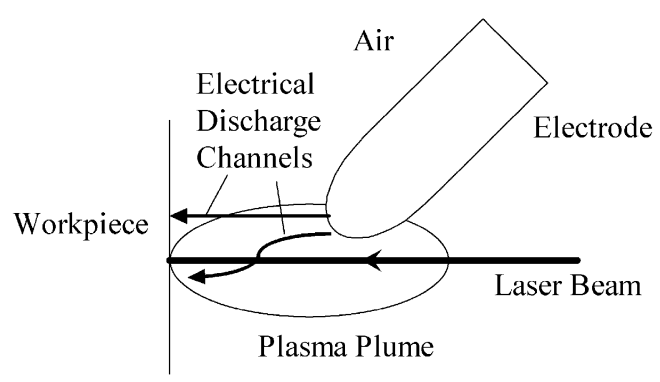

Fig. 10. Sketch of multi-channel forming of laser-induced discharge. 


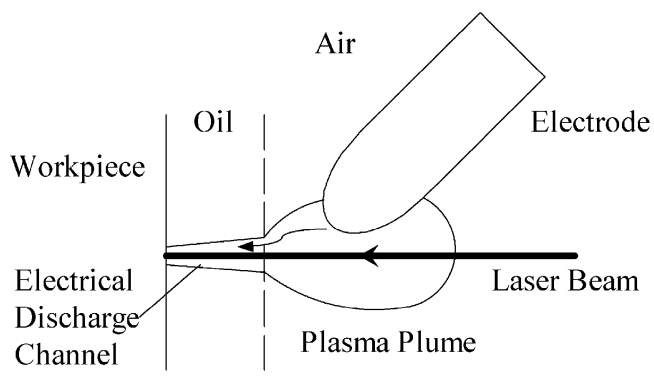

Fig. 11. Sketch of single channel forming of laser-induced discharge.

metal within the spot is melted and overheated. When the electrical pulse is shut off, the discharge ends suddenly, the pressure on the spot disappears, and the overheated liquid metal is shot out at once. Therefore a single-crater is formed, it is deep and its circumference is clear and regular.

\subsection{Laser spot coincident without crater and the differential diameters of crater}

The phenomena of laser spot coincident without crater and the differential diameters of crater can also be explained by the mechanism mentioned above. If the roll is not covered by a thin oil film, laser trigged plasma plume is in air, streamers are able to develop anywhere in the plasma. The discharge channel may be coincident with or without the laser beam. If it is not coincident, the discharge crater and the laser spot are separated, the latter is left and can be observed after discharging. If it is coincident, the spot is overlapped by discharge crater. When the rolls are covered by a thin oil film, the probability of channel formation decreases sharply, the streamers developing along the laser beam most likely result in discharge channels.

Since the channel expanding and the spot moving are restricted strongly by the oil film, diameters of crater with oil film are always smaller than that of without oil film apparently if other conditions are the same. It is concluded that covering a thin film may increase the energy density of channel when discharge energy is the same.

Therefore if the deterministic pattern is wanted, the roll must be anode and with an oil film. If the random pattern is wanted, the laser spot coincident without discharge crater is not a key problem, the roll being cathode in air may be better since larger diameters of crater improve the processing efficiency.

\section{Conclusions}

(1) For steel, iron and brass, the crater morphology in different polarities, waveforms and media are multicrater structures except the crater on anode with oil film.

(2) The diameters of crater with oil film are always smaller than that of without oil film when pulse energy, electrical polarity and current waveform are the same.

(3) The mechanism of multi-crater formation is multichannel discharging and plasma oscillation.

(4) Current waveforms have little effect on crater morphology.

(5) Both deterministic and random patterns can be obtained by the YAG laser-induced discharge texturing.

\section{Acknowledgement}

The authors would like to express their gratitude to Natural Science Foundation of China (Grant No. 60278023).

\section{References}

[1] K. Nishimura, N. Soeda, S. Ujihara, M. Nagaoka, Iron Steel Eng. 68 (1991 (Aug.)) 46.

[2] S. Ujihara, T. Hisatsune, Y. Hishida, M. Imanaka, H. Abe, Iron Steel Eng. 68 (1991 (Aug.)) 52.

[3] W.J. Davies, Iron Steelmak. 11 (1984 (March)) 37.

[4] J. Crahay, A. Bragard, Pro. 12th Bienn. Congr. of the International Deep Drawing Research Group, S. Margherita, Ligure, May 1982, La Metalluria Italiana, Milan, 1982, p. 153.

[5] M.F. El-Menshawy, M.S. Ahmed, 1985 SME Manufacturing Engineering Transactions: 13th NAMRC North American Manufacturing Research Conference Proceedings, Berkeley, California, USA, 1985, p. 470 .

[6] J. Dolves, Iron Steel Eng. 68 (1991 (Aug.)) 33.

[7] Yang Ming-Jiang, Chen Guan-Nan, et al., (1992). China Patent No. 92113223.9 (Chinese).

[8] Chen Guan-Nan, Yang Ming-Jiang, et al., (1993). China Patent No. 93109710.X (Chinese).

[9] Wang Hong-Cai and Yang Ming-Jiang, (2000). China Patent No. 00128273.5 (Chinese).

[10] Chen Guang-Nan, Appl. Laser 16 (1996) 155 (Chinese).

[11] O. Deutscger, Iron Steel Eng. 74 (1997 (May)) 35.

[12] Liu Ying, Chen Darong, Yang Wenyan, Chinese J. Mech. Eng. 39 (7) (2003) 107 (Chinese).

[13] A. Kovalchenko, O. Ajayi, A. Erdemir, G. Fenske, I. Etsion, Tribol. Trans. 47 (2004) 299.

[14] R.A. Dougal, P.F. Williams, J. Phys., D, Appl. Phys. 17 (1984) 903.

[15] S. Yoshida, S. Kubodera, T. Sakai, et al., J. Appl. Phys. 59 (6) (1986) 1904.

[16] Y.E. E-D. Gamal, N.M. Abdel-Moneim, J. Phys., D, Appl. Phys. 20 (1987) 757.

[17] R.M. Gilgenbach, Rev. Sci. Instrum. 54 (1) (1983) 109.

[18] Y. Hoshi, H. Yoshida, Y. Tsutsui, IEEE Trans. Plasma Sci. 28 (5) (2000) 1771.

[19] E.M. Bazelyan, Yu. P. Raizer, Spark Discharge, CRC Press, New York, 1998, p. 8.

[20] E. Sher, J. Ben-Ya'Ish, T. Kravchik, Combust. Flame 89 (1992) 186.

[21] Pan Yongjiang, Hu Chuanjin, in: Institute of Electrical Engineering Chinese Academy of Sciences (Ed.), Proceedings of Institute of Electrical Engineering Chinese Academy of Sciences, Beijiing, vol. 15, 1988, p. 96 (Chinese).

[22] Qi Li-wei, Lou Le-ming, Li Ming-hui.Mechanism of electrical discharge machining and wave characteristics of plasma channel. J. of Shanghai Jiaotong University, 35 (7) (2001) 989 (Chinese). 